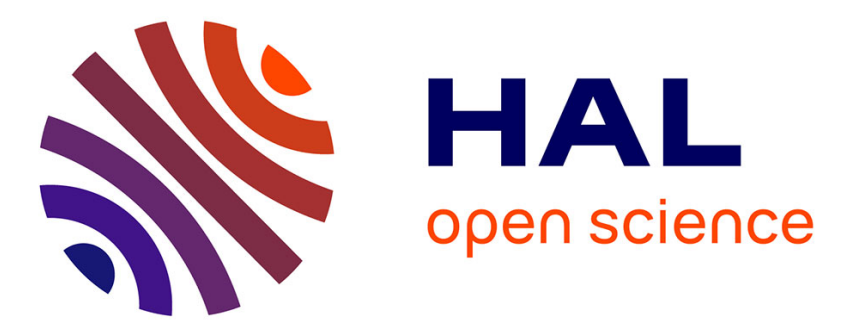

\title{
Probing fluid torque with a hydrodynamical trap: Rotation of chiral particles levitating in a turbulent jet
}

Thomas Barois, Peter D Huck, Charles Paleo, Mickaël Bourgoin, Romain Volk

\section{To cite this version:}

Thomas Barois, Peter D Huck, Charles Paleo, Mickaël Bourgoin, Romain Volk. Probing fluid torque with a hydrodynamical trap: Rotation of chiral particles levitating in a turbulent jet. Physics of Fluids, 2019, 31 (12), pp.125116. 10.1063/1.5131207 . hal-02427609

\section{HAL Id: hal-02427609 \\ https://hal.science/hal-02427609}

Submitted on 3 Jan 2020

HAL is a multi-disciplinary open access archive for the deposit and dissemination of scientific research documents, whether they are published or not. The documents may come from teaching and research institutions in France or abroad, or from public or private research centers.
L'archive ouverte pluridisciplinaire $\mathbf{H A L}$, est destinée au dépôt et à la diffusion de documents scientifiques de niveau recherche, publiés ou non, émanant des établissements d'enseignement et de recherche français ou étrangers, des laboratoires publics ou privés. 


\title{
Probing fluid torque with a hydrodynamical trap: rotation of chiral particles levitating in a turbulent jet
}

\author{
T. Barois, ${ }^{1}$ P. D. Huck, ${ }^{2}$ C. Paleo, ${ }^{2}$ M. Bourgoin, ${ }^{2}$ and R. Volk ${ }^{2}$ \\ 1) Univ. Bordeaux, CNRS, LOMA, UMR 5798, F-33405 Talence, France \\ ${ }^{2)}$ Laboratoire de Physique, École Normale Supérieure de Lyon, Université de Lyon, CNRS, 46 Allée d'Italie, \\ F-69364 Lyon, cedex 07, France
}

(Dated: 3 January 2020)

A vertical turbulent jet is used to trap chiral particles. The particles are maintained in levitation and a stationary rotation regime is observed. The model particles used are composed of a sphere and a helical tail. The rotating performance of the particles is investigated as a function of the length and the twisting of their tails. In addition, the flow field around a spherical particle trapped in the jet is characterized by a particle tracking velocimetry technique (3D-PTV). This flow characterization is used to compute the nearfield velocity around a captured particle and to predict the rotation reported for the different geometries tested.

PACS numbers: 47.27.wg, 45.20.dc, 47.80.Cb

\section{INTRODUCTION}

Wind tunnels measurement is probably the first strategy ${ }^{1,2}$ on could think of in order to study the relation between flows and forces. These facilities may be used to measure drag, lift or torque on test bodies in various fields such as aeronautics, ballistics or biomechanics. The measure is performed by placing an object in a controlled upstream flow and measuring the aerodynamic force components acting on the model body. In this approach, the quality of the measure depends on the quality of the upstream flow.

A second strategy to measure fluid forces on an object is towing ${ }^{3}$. The point of towing is to have an object moving in a fluid perfectly at rest without the need to control the flow as in wind tunnels. The drawback of the towing strategy is that the measurement apparatus has to move with the object and stationarity is possible as long as constant towing speed is assured.

The object of this article is to consider fluid levitation by a turbulent jet a third strategy to investigate the fluid forces acting on the levitating object.

A stationary levitation is observed when a sphere is placed in the flow of a vertical or tilted turbulent jet ${ }^{4-8}$. For a range of parameters, the sphere is trapped by the jet and it fluctuates around a stable equilibrium position where the gravitational force and the mean fluid forces are balanced. The levitation of a sphere in a turbulent jet shares a similarity with the free settling of a sphere ${ }^{9}$ since there is an equilibrium with the gravitational force and either the object or the fluid is in motion. Note however that the levitation in a turbulent jet and the free settling of a sphere are not equivalent: first, the flow profile of such jets is not spatially uniform in jet, and second, the intrinsic velocity fluctuations in a turbulent jet may modify the value of the drag and lift coefficient on the sphere $\mathrm{e}^{10,11}$.

A turbulent jet is obtained with a fluid injected through a nozzle at a sufficiently high velocity. Except close to the nozzle, the structure of a turbulent jet is universal and does not depend on the specific shape of the nozzle. Universality is an interesting property here because the turbulent jet configuration can be considered a reference flow easy to reproduce.

The levitation of an object in a fluid is a situation with a stationary regime for a particle in a flow. Stationarity is convenient from an experimental point of view because it permits averaging of physical observables. Contrary to wind tunnel experiments with attached test bodies, levitation experiments are situations where the net fluid force is directly inferred from the balance with the gravitational force. Consequently, the investigation of fluid forces in a levitation experiment transfers to the investigation of the velocity field around the trapped particle. This can be done either by performing time and space resolved flow measurements or by using a calibration of the jet flow profile and a measurement of the location and orientation of the trapped particle. It should be mentioned that free particles can also be studied in quasi-stationary levitation with a vertical wind tunnel if the velocity is chosen to be the settling velocity of the object ${ }^{12-14}$.

For a proof of the relevance of the levitation technique, we consider a model situation where we study the relation between the chirality of an object of simple geometry and its rotation rate when it is levitating. The fluid levitation strategy is particularly adapted for rotation studies because it is a simple way to position an object in a flow while permitting free rotation by avoiding the use of mounting supports. A similar situation is possible in simulations ${ }^{15}$ in which the position of the sphere can be rigorously imposed independently of the orientation of the sphere.

Chiral particles in relation to their spinning is studied for toy projectiles ${ }^{16,17}$ and some types of plants seeds ${ }^{18}$. Concerning chiral particles in upstream flows, a geometry of chiral discs have been recently studied ${ }^{19}$ to explore the individual and the collective dynamics of active rotors in quasi 2D experiments. Active rotors are a class of 
(a)

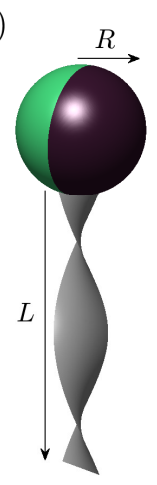

(b)

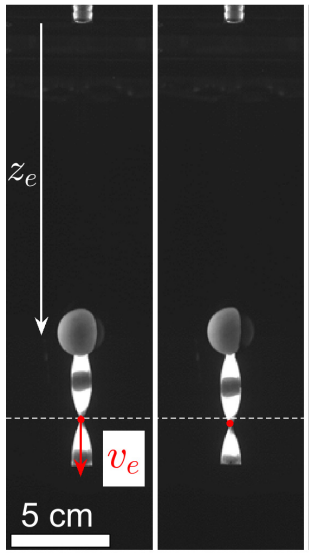

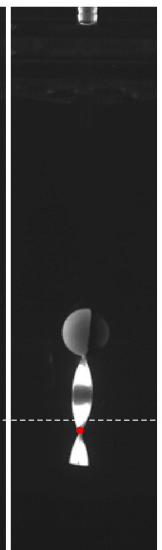
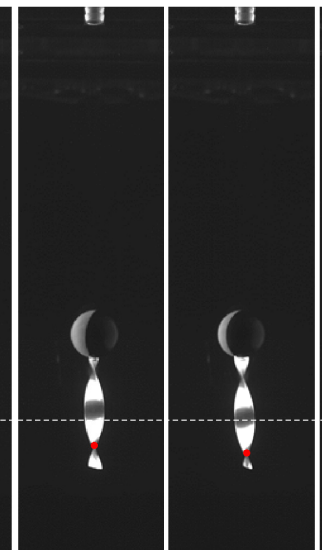

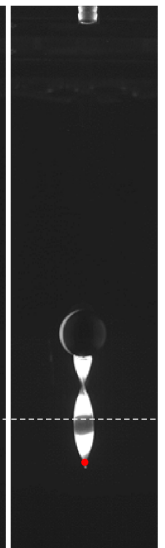

(c)

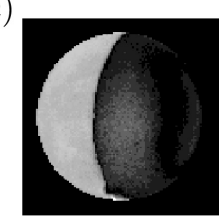

(d)

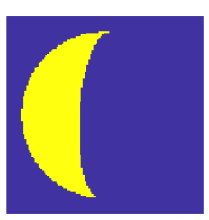

FIG. 1. a) Schematics of a chiral particle. The sphere has a radius $R$ and the twisted tail has a length $L$. b) Image sequence of a chiral particle in stationary rotation trapped in a turbulent water jet with $R=12 \mathrm{~mm}$ and $L=58 \mathrm{~mm}$. The particle is buoyant and it is trapped at a distance $z_{e}$ to the nozzle of the jet visible at the top of the images. The time delay between frames is $200 \mathrm{~ms}$. A constant flow rate $\left(Q=2.2 \times 10^{-5} \mathrm{~m}^{3} \cdot \mathrm{s}^{-1}\right)$ is injected from the nozzle. $z_{e} / R=13.4$. The phase velocity of the tail $v_{e}$ corresponds to the velocity of the node indicated by a red dot. A horizontal dashed line is added to visualize the phase evolution c) Image centered on the sphere of the chiral particle d) Synthetic image used in the pattern analysis to determine the orientation of the particle.

active particles with experimental realizations and models mostly in the low Reynolds number limit ${ }^{20-24}$. The strategy with chiral particles in levitation presented here would be beneficial to investigate active rotors in $3 \mathrm{D}$ configurations for high Reynolds number regimes.

The first part (II) of this article describes the experimental setup in which a turbulent jet is used to trap a chiral object. A stationary trapping is observed and the chiral particles in levitation are rotating. In the second part (III), the relation between chirality and rotation speed is measured. The flow around a reference spherical particle is studied in the third part (IV). The flow profile measured in this configuration is used in the final section of the article $(\mathrm{V})$ to establish a predictive model for the rotation of such model particles.

\section{EXPERIMENTAL SETUP}

An immersed water jet is set in a tank of $30 \times 30 \times$ $70 \mathrm{~cm}^{3}$. The jet is vertical and pointing downward. A closed-circuit is used to inject water through a circular nozzle with an internal diameter of $6.5 \mathrm{~mm}$. The exit of the nozzle is located 2 centimeters below the water surface. The water is collected at the bottom of the tank with a similar nozzle placed in the center. This immersed turbulent jet is used to capture a buoyant sphere, with or without an added tail, in a stable levitation state. The sphere used in the whole set of experiments is made of polypropylene with a mass density of $\rho_{b}=846 \mathrm{~kg} \cdot \mathrm{m}^{-3}$ and a radius $R=12 \mathrm{~mm}$. A view of the nozzle with a captured particle is given in the chronophotography in figure $1 \mathrm{~b}$ ).

The stability of a sphere in the plane perpendicular to the jet axis is related to the Coandă effect ${ }^{25,26}$. The Coandă effect relates to a transverse force exerted on curved surfaces: if the sphere is slightly displaced from the jet axis, the jet is deflected from the vertical direction and the momentum conservation results in a net attractive force for the sphere towards the jet axis. The Coandă effect involves the fluid inertial transfer and the fluid levitation in vertical jets ${ }^{5}$ is observed for high Reynolds numbers in a regime for which the jet is necessarily turbulent. The force landscape due to the Coandă effect depends on the geometry of the object: the stationary levitation is not observed for simple shapes such as cylinders or cubes.

The chiral particles studied are obtained by adding a helical tail to the polypropylene sphere. A representation of the particle geometry is given in figure 1 a). The tails used in the experiments are obtained from the mechanical twisting of aluminum plates $\left(\rho_{t}=2700 \mathrm{~kg} . \mathrm{m}^{-3}\right)$ first cut in ribbons and then twisted with hand tweezers. The twisting by the hand tweezers is irreversible and the tails are rigid during the experiments. In order to quantify the twist of the tail, a twisting wavenumber $h=\Delta \theta / L$ is defined where $\Delta \theta$ is the cumulative angle between the angle of the tail at the attachment with the sphere and the angle of the tail at the free end at the distance $L$. The twisting wavenumber $h$ also applies locally since the tails are uniformly twisted. The same thickness $(e=1 \mathrm{~mm})$ and width $(W=10 \mathrm{~mm})$ are used for the different tails. The lengths of the tail for the 5 specimens studied are $L=14,20,30,42$ and $58 \mathrm{~mm}$. All the specimens studied are buoyant. 
A camera is used to record the free rotation of chiral particles trapped in the turbulent jet. The particle is illuminated with two light screens placed at each side of the water tank.

The chiral particles are manually placed in the jet. If the free surface is at rest, a particle is in a steady rotation state. Figure $1 \mathrm{~b}$ ) shows an image sequence of a rotating particle in the turbulent jet $(L=58 \mathrm{~mm}$, time stamp 200 $\left.\mathrm{ms}, Q=2.2 \times 10^{-5} \mathrm{~m}^{3} \cdot \mathrm{s}^{-1}\right)$. The flow rate is such as the particle is trapped in the center of the view field of the camera. For the rotation measurements, the distance to nozzle is $z_{e}=13.4 \times R=160 \mathrm{~mm}$.

The orientation of the sphere is extracted from a pattern analysis. Here, the pattern is a hemisphere with half of the sphere is painted in black (figure $1 \mathrm{c}$ ) and d)). A more complex pattern would give access to the full orientation of the particle ${ }^{27}$. Here, only the phase, or equivalently the azimuth angle, $\psi$ with respect to the particle axis is extracted which is sufficient to determine the angular velocity of the particle along its axis. The polar angle could be also obtain in principle from the analysis of the orientation of the tail with respect to the vertical.

Although the chiral particles are stationary trapped, both the position and the orientation are fluctuating. The orientation of the tail is aligned with the gravity field on average but the turbulent fluctuations are forcing a swinging motion of the tail of a typical angle with the vertical of $0.2 \mathrm{rad}$. The associated variation of the vertical component of the velocity is $1-\cos 0.2 \approx 2 \%$.

\section{ROTATION OF A LEVITATING CHIRAL PARTICLE}

The relation between the rotation of a chiral particle and the twist of its tail has been studied for the 5 specimens that were described in section II. In this section, only the specimen with the $30 \mathrm{~mm}$ tail is presented.

To study the influence of the twisting wavenumber, the tail has been progressively twisted from $h=0 \mathrm{rad} . \mathrm{m}^{-1}$ (flat tail) to a maximal twisting of $h=96 \mathrm{rad} . \mathrm{m}^{-1}$. The twist angle of the tail is measured from image analysis of a picture of the tail from a bottom view (not shown here).

The equilibrium distance to the nozzle is found to be weakly affected by the twisting wavenumber. The relative variation for $z_{e}$ is $3 \%$ between $h=7$ rad.m ${ }^{-1}$ and $h=76$ rad. $\mathrm{m}^{-1}$.

The orientation of the trapped particle $\psi(t)$ is represented in figure 2 for 5 values of the twisting wavenumber. The angular frequency is computed from the angle increment over the 20 seconds of the sequence.

Figure 3 shows the spinning angular frequency $\omega$ of the trapped particle as a function of the twisting wavenumber $h$. The rotation speed of the free particle is found to be proportional to the twist of the tail. The adjustment of the data points is represented where $\omega_{h}=h v_{e}$ with a slope $v_{e}=4.0 \times 10^{-2} \mathrm{~m} . \mathrm{s}^{-1}$ with a physical dimension of

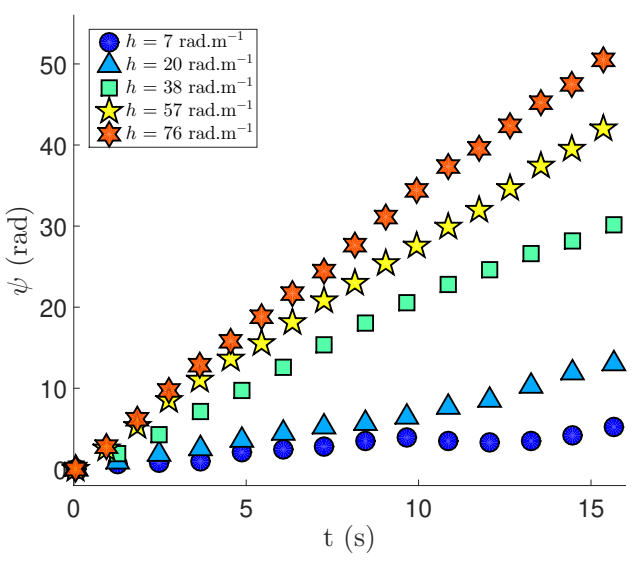

FIG. 2. Azimuth angle (phase) $\psi(t)$ of a chiral particle trapped in a turbulent jet as a function of time. The instantaneous angular frequency of the rotating particle is $d \psi / d t$. Five chiralities are presented where $h=7,20,38,57$ and 76 rad. $\mathrm{m}^{-1}$ are the twisting wavenumbers of the particle's tail. The length of the tail is $L=30 \mathrm{~mm}$ and the flow rate of the jet is $Q=4.3 \times 10^{-5} \mathrm{~m}^{3} \cdot \mathrm{s}^{-1}$ for an average equilibrium distance to the nozzle $z_{e}=140 \mathrm{~mm}$.

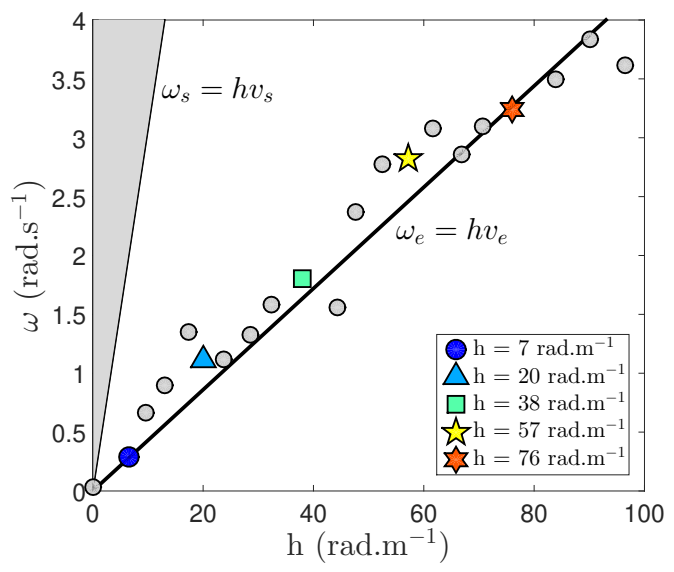

FIG. 3. Average angular velocity $\omega=\langle d \psi / d t\rangle$ as a function of the twisting wavenumber $h$ of the tail of length $L=30 \mathrm{~mm}$. The solid line marking the frontier with the gray area is the no-slip limit $\omega_{s}=h v_{s}$ with $v_{s}=2.3 \times 10^{-1} \mathrm{~m} . \mathrm{s}^{-1}$ for which the phase velocity of the tail equals the fluid velocity. The 21 data points are adjusted with a second solid line $\omega_{e}=h v_{e}$ with $v_{e}=4.0 \times 10^{-2} \mathrm{~m} . \mathrm{s}^{-1}$. The ratio $\omega_{e} / \omega_{s}$, or equivalently $v_{e} / v_{s} \sim 0.17$, represents the efficiency of the rotation. The twisting wavenumbers $h=7,20,38,57,76 \mathrm{rad} . \mathrm{m}^{-1}$ are represented with different symbols and they correspond to the time evolution presented in figure 2 with the same symbols.

a velocity. This velocity corresponds the phase velocity of the travelling wave of the tail. The phase velocity is represented in the chronophotography in figure $1 \mathrm{~b}$. It corresponds to the velocity of the node of the tail indi- 
cated by a red spot. The linear relation between $h$ and $\omega$ is interesting here because it is possible to infer the twist of a chiral particle from the measurement of $\omega$.

The velocity $v_{e}$ is compared to the settling velocity of the particle $v_{s}$ computed from the balance between weight and drag in a uniform flow:

$$
\left(\frac{4}{3} \pi R^{3}\left|\rho-\rho_{b}\right|-L W e\left|\rho-\rho_{t}\right|\right) g=\frac{1}{2} c_{D} \rho \pi R^{2} v_{s}^{2}
$$

where $\rho$ is the density of the fluid, $\rho_{b}$ the density of the sphere, $\rho_{t}$ the density of the tail, $L W e$ the volume of the tail, $R$ the sphere radius, $g$ the gravitational field and $c_{D}$ the drag coefficient of the particle. The drag coefficient of the particle with a tail is assumed to be that of the sphere only with $c_{D}=0.5$. The obtained value from equation (1) is $v_{s}=2.3 \times 10^{-1} \mathrm{~m} . \mathrm{s}^{-1}$.

It should be expected that the adding of a tail to the spherical particle would modify the drag coefficient ${ }^{28}$. However, the modification of the drag coefficient should be reasonably small here because the sphere cross section $\pi R^{2}=452 \mathrm{~mm}^{2}$ is much larger than the splitter cross section $t W=10 \mathrm{~mm}^{2}$ and, additionally, the splitter is shielded by the sphere. Besides, the value for $v_{s}$ is only used here as a scaling velocity to be compared with the characteristic velocity $v_{e}$. Last, numerical simulations for similar geometries with spheres ${ }^{29}$ or cylinders ${ }^{30,31}$ show that the presence of a flat appendage modifies the drag coefficient by $1-3 \%$ percents typically.

No experimental data points are found in the region above the line $\omega_{s}=h v_{s}$ in figure 3 . The line $\omega_{s}=h v_{s}$ corresponds to a no-slip regime for which the phase velocity of the tail $v_{t}=\omega / h$ equals the settling velocity $v_{s}$. In this limit, there is no fluid torque on the tail since there is no relative velocity between the fluid and the surface of the tail.

An optimization problem can be addressed here with the tail length that maximizes the rotation for a trapped particle. To address this problem, the rotation has been investigated for the specimens obtained with the same sphere and the 5 tails of different lengths. The sphere has a radius $R=12 \mathrm{~mm}$ and the different tails correspond to normalized lengths $L / R=1.2,1.7,2.5,3.5$ and 4.8. $L / R=2.5$ corresponds to the particle presented in detail previously with the experimental results in figures 2 and 3. Because the rotation speed is proportional to $h$, an efficiency independent of $h$ is defined with the ratio of the rotation regime $\omega_{e}=h v_{e}$ by the optimal rotation regime $\omega_{s}=h v_{s}$ :

$$
\eta=\frac{\omega_{e}}{\omega_{s}}=\frac{v_{e}}{v_{s}}
$$

where $\eta$ quantifies a slip velocity between the phase velocity of the tail $v_{e}$ and the typical velocity of the fluid $v_{s} . \quad v_{e}$ is estimated from the proportional adjustment of $\omega$ as a function of $h$. Figure 3 is presenting a solid line corresponding to the adjustment for the data point $L / R=2.5$. The settling velocity $v_{s}$ is computed using equation (1).

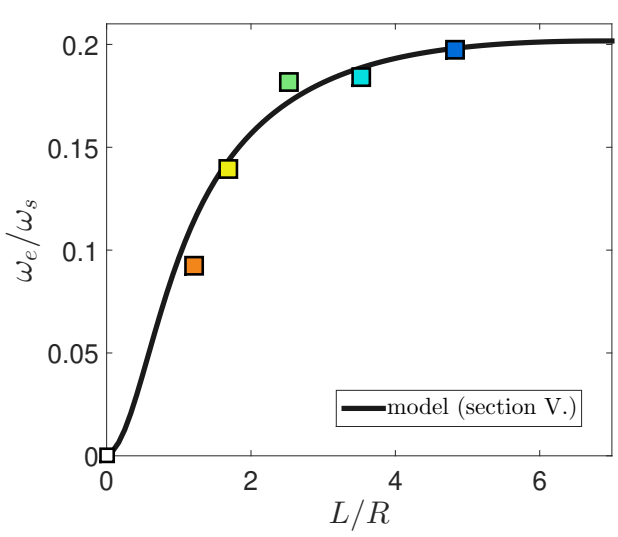

FIG. 4. Rotation efficiency $\eta=\omega_{e} / \omega_{s}$ as a function of the tail relative length $L / R$. Each data point corresponds to a set of experiments where the twist of a tail of length $L$ is

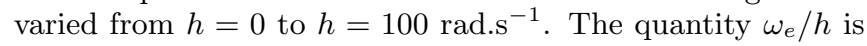
estimated from the linear adjustment of the angular rotation frequency measured as a function of the twisting wavenumber (see figure 3 for the data point for $L=30 \mathrm{~mm}$ ). The solid line is the angular rotation predicted by the model discussed in section $\mathrm{V}$ with a single adjustable parameter.

The rotation performances of particles with different tail's length is presented in figure 4 . It shows that the length of the tail has to be at least twice the radius of the sphere to approach an optimal regime with $\eta$ of the order of 0.2. Considering our particles to be simplified models of chiral projectiles, this may explain for instance why certain toys, such as footballs with chiral tails ${ }^{16,17}$ or plant seeds ${ }^{18}$, are specifically designed to avoid placing the tail in the close proximity of the body.

\section{FLOW AROUND A TRAPPED SPHERE}

In this section, the flow around a trapped sphere in the turbulent jet is investigated. The measured flow will be used to establish an empirical formulation for the mean velocity in the wake of the sphere without a tail. The expression for the wake velocity profile will then be used to predict the forcing torque when a twisted tail is attached to the sphere (section V).

The velocity profile of the jet in the presence of the sphere is characterized by a particle tracking velocimetry technique $(\mathrm{PTV})^{32}$. Fluid flow measurement techniques, such as PTV measurements, are particularly challenging here because of the presence of walls ${ }^{33}$ with the sphere surface, the fluctuation of the sphere location and the spatial non-homogeneity of the tracers because of the seeding procedure. The polystyrene tracing particles (diameter 500-800 $\mu \mathrm{m}$ ) are injected through the nozzle while the turbulent jet is established which means that the flow is mapped only for the fluid that comes from the nozzle. The tracing particles have a mass density of $1004 \mathrm{~kg} \cdot \mathrm{m}^{-3}$. 
The tracing particles paths are individually recorded with two cameras and stereoscopic reconstruction is used to determine the 3D coordinates of each particle for each frame. The injected flow rate is $4 \times 10^{-5} \mathrm{~m}^{3} \cdot \mathrm{s}^{-1}$ which corresponds to a typical velocity of $1.2 \mathrm{~m} . \mathrm{s}^{-1}$ at the nozzle. This specific flow rate was chosen so that the particle in levitation is in the center of the camera field in order to simplify the pattern recognition analysis. The sequence has a duration of $47 \mathrm{~s}$ with a frame rate of $340 \mathrm{~Hz}$.

The trajectories of the tracing particles are used to construct Eulerian mappings of the velocity field. A cylindrical binning grid is used with a mesh resolution of 1.5 $\mathrm{mm}$ for both the radial and the axial coordinates.

Two representations of the velocity fields are proposed : (i) in the laboratory's frame and (ii) in the sphere's frame. In both representations, the cylindrical coordinates $(r, \theta, z)$ are used where $z$ corresponds to the jet axis and the symmetry of revolution is assumed (invariance with respect to $\theta$ ). The $z$-axis points downward in order to have the distance to the nozzle counted positively.

The vertical component of the mean velocity for the jet with a trapped sphere is represented in figure 5 (a) in a logarithmic color scaling. The representation in the nozzle frame is well adapted to study the scattering of the jet by the sphere. The structure of a free turbulent jet is considered to be universal ${ }^{34-36}$ but there is not a unique reference formulation for the velocity profile. Here, a Gaussian approximation ${ }^{37-43}$ is used as a fitting function for the jet mean velocity field:

$$
v_{j}(r, z)=\frac{D}{z-z_{0}} \exp \left(-k_{u}\left(\frac{r}{z-z_{0}}\right)^{2}\right)
$$

where $z_{0}$ is the virtual origin of the jet and $D$ is homogeneous to a diffusion coefficient defining the magnitude of the jet velocity. $k_{u}$ is a dimensionless parameter that relates to the opening angle $\alpha$ of the jet with $\alpha \propto 1 / \sqrt{k_{u}}$. The validity of the Gaussian jet approximation for our specific setup can be found in a previous work ${ }^{44}$ for the same turbulent jet without trapped sphere.

Although the Gaussian approximation is valid for the jet without the trapped sphere, the Gaussian profile (3) is also used here to analyse the velocity profile of the jet in the presence of the sphere. Because a turbulent jet is a self-established shear flow, we expect that the Gaussian profile is robust away from the fluid boundaries. As an example, this is verified for free turbulent jets for which the details of the injecting nozzle does not influence the jet structure after few nozzle diameters.

Two sets of parameters for the jet profile (3) are adjusted with the measured profile. The optimized fitting parameters $D, k_{u}$ and $z_{0}$ are presented in table I. The first adjustment is for the flow upstream to the ball and the corresponding flow profile is presented in figure 5 (b). The second adjustment is performed for the region downstream to the ball and and presented in figure 5 (c). The parameter $k_{u}$ is usually in the range 55 to 100 for open jets $^{43}$. Here, $k_{u}$ is larger than 100 in the upstream region which mean that the opening angle of the jet is smaller than expected. This large value of $k_{u}$ could be attributed to the close proximity of the nozzle and the fact that the jet self-similar profile is not fully established to close from the nozzle. Also, the adjustment for the region upstream to the ball is performed on a narrow region. In the region downstream to the ball, the parameter $k_{u}$ is slightly smaller than the values reported for open jets. This means that the opening angle of the jet at a distance $z_{e}+3 R<z<z_{e}+5 R$ is slightly higher than usual which is not surprising here because the free jet profile is scattered by the sphere. The virtual origin of the jet is also shifted by $10.6 \mathrm{~mm}$ between the upstream and the downstream profiles. The virtual origin is the source point of the equivalent jet profile. The virtual origins are all negative which means that they are located slightly above the images in the figures 5 and 6 .

The flow field is then investigated in the reference frame of the sphere's center. This representation is relevant to address the flow from the perspective of the fluctuating sphere. More specifically, the flow in the near wake of the sphere will be used to estimate the net torque when a twisted tail is added to the sphere.

The trajectory of the trapped sphere is extracted from the recordings. The mean location of the trapped sphere is represented in figure 5 (a) by a circle. The equilibrium trapping distance to the nozzle is $z_{e}=70 \mathrm{~mm}$ which corresponds to 5.8 sphere radii. Since the sphere is fluctuating around its equilibrium position, the spatial sampling of the flow is not strictly restricted to the excluded volume of the sphere at its average position. The standard deviation is computed from the track of the sphere where $\Delta x_{e}=0.67 \mathrm{~mm}, \Delta y_{e}=0.64 \mathrm{~mm}$ and $\Delta z_{e}=1.83 \mathrm{~mm}$. The maximal excursion of the sphere is typically 5 times the standard deviation in the corresponding direction.

Figure 6 (a) is a representation of the mean velocity flow field in the frame of the trapped sphere. The velocity field in the sphere's frame is obtained in two steps. First, and for each time step, the coordinates of the trapped sphere is subtracted to the coordinates of the tracing particles. Second, the trajectories of the tracing particle in the sphere frame are processed to estimate the velocity of the flow. A cylindrical binning grid is used to obtain a Eulerian representation of the velocity. In this representation, the location of the nozzle is a fluctuating quantity.

The fitting profile is adjusted to the measured flow in both upstream and downstream regions (dashed boxes in figure 6 (b) and (c), table I). The model profile obtained is used to compute the relative variation of the velocity $\sigma_{v}$ due to the presence of the ball. This variation is defined by

$$
\sigma_{v}=\frac{v_{z}-v_{j}}{v_{j}}
$$

where $v_{z}$ is the vertical component of the velocity measured in the sphere's frame and $v_{j}$ is the model flow profile (3) for turbulent jets with the adjustable parameters 
(a) $3 \mathrm{~cm}$

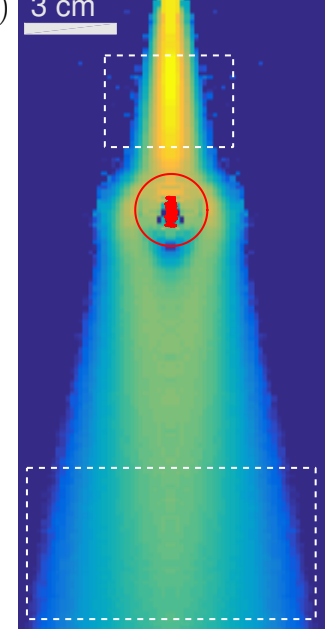

(b)

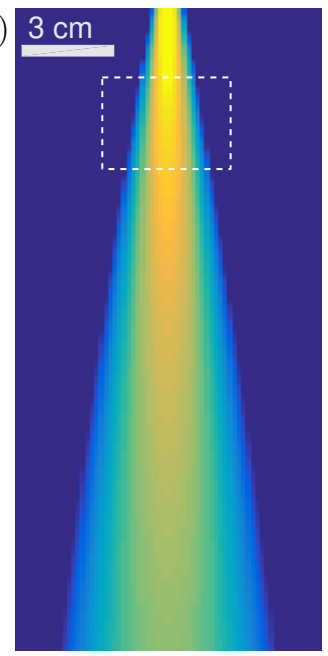

(c) $3 \mathrm{~cm}$

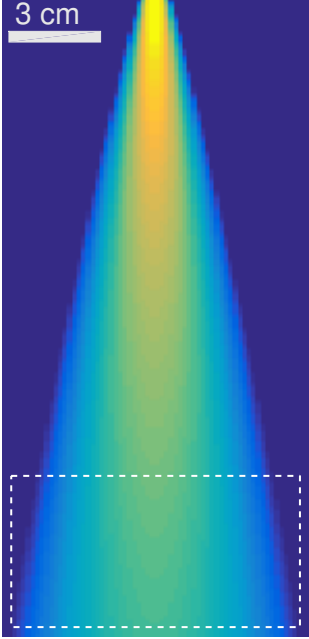

FIG. 5. (a) Vertical component of the mean velocity profile measured in the frame of the nozzle. The circle indicates the mean position of the trapped sphere fluctuating in the jet. The trajectory path of the sphere center is also indicated and it covers a vertically elongated region inside the added circle. The fluctuations of the sphere are of the order of its radius in the vertical direction and of its half-radius in the equatorial plane. (b) and (c) are the estimated upstream and downstream profiles using equation (3). The dashed boxes indicate which portion of the measured flow was used to adjust the fitting parameters. The velocity is represented in a logarithmic color scaling for the three velocity fields.

(a)

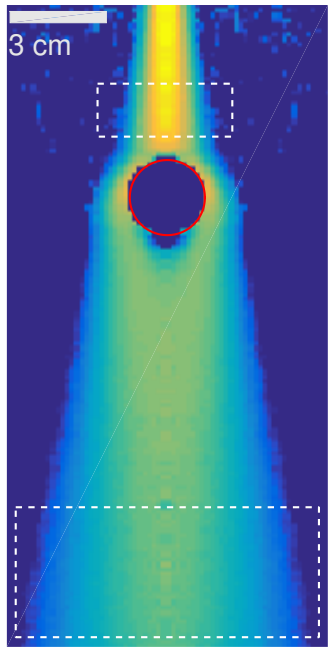

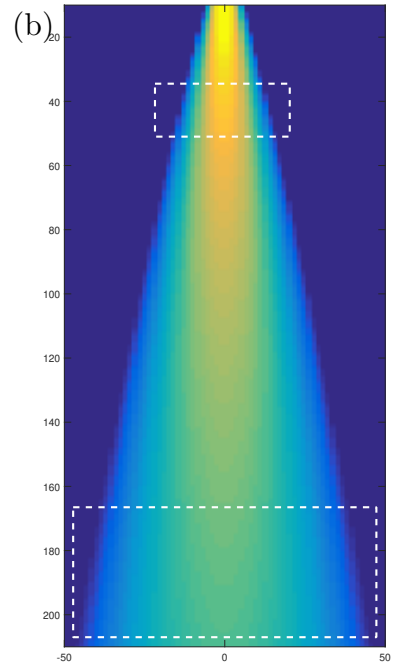

FIG. 6. (a) Vertical component of the mean velocity profile $v_{z}$ measured in the frame of the trapped ball. The velocity is represented in a logarithmic color scaling. The circle indicates the position of the trapped sphere. (b) Estimated flow profile using an adjustment of the fitting function $v_{j}$ from equation (3). The adjustment is for the data points in the two dashed boxes. The velocity is represented in a logarithmic color scaling. 


\begin{tabular}{|c|c|c|c|c|c|c|}
\hline reference frame & region of interest & $D\left(\mathrm{~m}^{2} \cdot \mathrm{s}^{-1}\right)$ & $k_{u}$ & $z_{0}(\mathrm{~mm})$ & figure & relative error \\
\hline nozzle & upstream & $6.6 \times 10^{-2}$ & 118.5 & -26.1 & $5(\mathrm{~b})$ & $12 \%$ \\
nozzle & downstream & $4.0 \times 10^{-2}$ & 50.3 & -15.5 & $5(\mathrm{c})$ & $3.7 \%$ \\
trapped sphere & upstream \& downstream & $4.2 \times 10^{-2}$ & 52.3 & -10.2 & $6(\mathrm{~b})$ & $5.0 \%^{*}$ \\
\hline
\end{tabular}

TABLE I. Adjustement parameters for the jet Gaussian approximation (Eq. (3)) in the upstream and downstream region. $D$ is the velocity magnitude of the jet, $k_{u}$ a dimensionless parameter and $z_{0}$ is the virtual origin. The relative error is the distance between the measured velocity and the Gaussian model normalized by the root mean square velocity. The star symbol $\left({ }^{*}\right)$ signals that the relative error is only computed in the downstream region for the velocity map in figure 6 .

presented in table I.

The relative velocity difference $\sigma_{v}$ is presented in figure 7 for different distances $z$ to the sphere center and also in figure 8 with a map representation. The modification of the flow due to the presence of the sphere reveals an axisymmetric mode where the velocity is reduced on the jet axis and increased in the equatorial region.

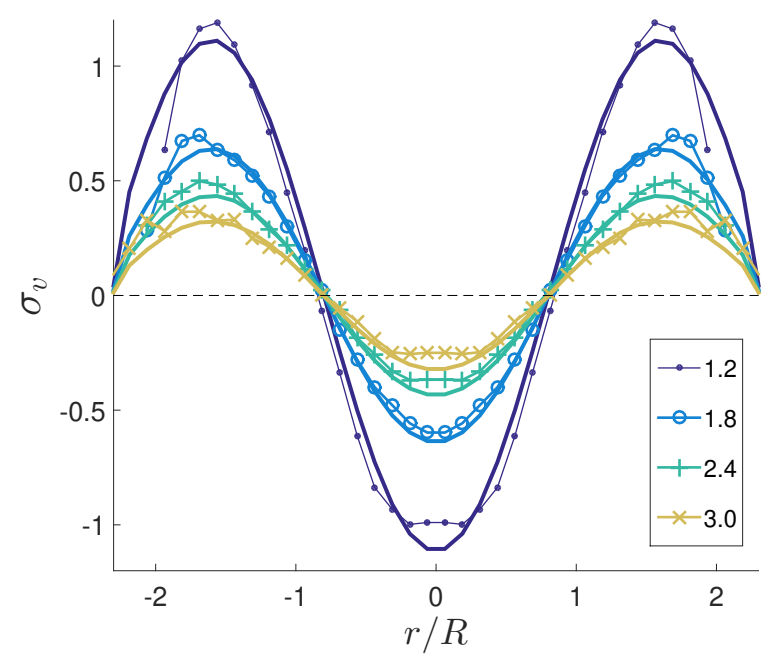

FIG. 7. Relative variation of velocity $\sigma_{v}=\left(v_{z}-v_{j}\right) / v_{j}$ computed in the wake of the sphere from the measured flow $v_{z}$ (Fig 6 a) and the Gaussian approximation for turbulent jets $v_{j}$ (Fig $6 \mathrm{~b}) . \sigma_{v}$ is represented a function of the normalized coordinate $r / R$ for 4 distances to the sphere $\left(z-z_{e}\right) / R=1.2,1.8,2.4$ and 3.0. A solid line is used to represented the empirical profile given in equation (5).

The wake of the sphere is investigated in more detail in figure 7 where the relative velocity variation $\sigma_{v}$ is presented as a function of the reduced radial coordinate $r / R$ for different distances to the sphere $\left(z-z_{e}\right) / R$. A self-similar profile for the relative velocity variation $\sigma_{v}$ is found where the amplitude of the oscillating mode decreases with the distance to the sphere $z-z_{e}$. The following empirical expression is proposed to account for the flow in the wake of the sphere:

$$
\sigma_{v}=A(\zeta) \cos \left(\frac{2 \pi}{\ell} \tilde{r}\right)
$$

where $\tilde{r}=r / R$ is the radial coordinate normalized by the sphere radius $R$ and $\zeta=\left(z-z_{e}\right) / R$ is the distance to the sphere normalized by $R . \ell$ is the dimensionless wavelength and $A$ is the magnitude of the velocity variation. The adjustment of the profile (5) to the experimental data is obtained with $\ell=3.2$ and $A=-1.36 \times \zeta^{-1.3}$. This fitting profile is presented as a solid line in figure 7 and as a field in the inserted image in figure 8 . The free parameters, $A$ and $\ell$, are adjusted using the analysis of the minima and maxima of the relative velocity variation represented in figure 7 .

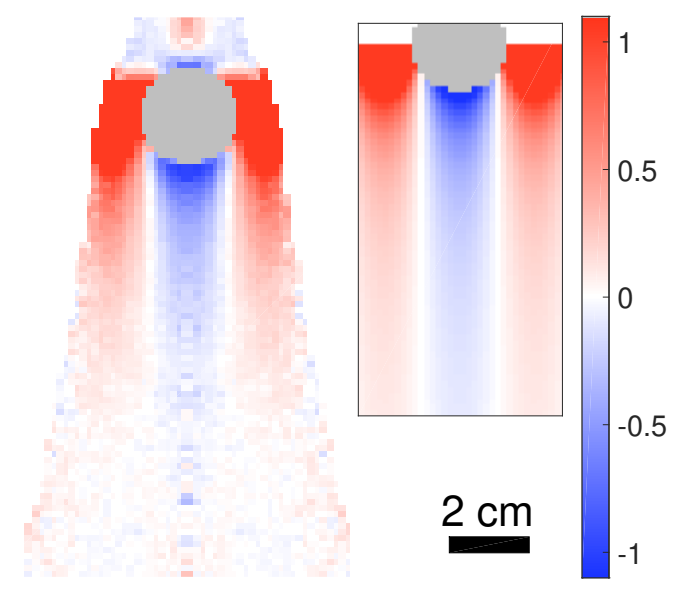

FIG. 8. Relative variation of velocity $\sigma_{v}=\left(v_{z}-v_{j}\right) / v_{j}$ around a trapped sphere at a distance $z_{e}=5.8 R$ of the nozzle with $v_{j}$ the Gaussian approximation for a turbulent jet and $v_{z}$ the measured flow. The inserted image is the empirical profile given in equation (5).

The relation (5) and the value of the free parameters $A, \zeta$ and $\ell$ have been established from the PTV measurements for the value $z_{e} / R=5.8$. The value $z_{e} / R$ was chosen to be rather small to have a large view of the region downstream to the ball. The formulation of equation (5) used rescaled parameters allowing an extrapolation of $\sigma_{v}$ for other values of $z_{e}$ and $R$ such as $z_{e} / R=13.4$ in the case of the rotation measurements. 


\section{MODEL FOR THE STATIONARY ROTATION}

The stationary rotation speed of the chiral particle is computed in this section by modeling the net torque. The torque on the chiral particle has two components, $\Gamma_{s}$, the damping torque exerted on the sphere, and $\Gamma_{t}$, the forcing torque applied to the helical tail.

The damping torque $\Gamma_{s}$ is due to the friction of the fluid exerted on the rotating particle. The friction stress on a surface element of the sphere is given by the Darcy-Weisbach equation with $\tau=(1 / 8) f \rho v_{t}^{2}$ where $f$ is a dimensionless friction factor and $v_{t}$ the tangential velocity. Here the tangential velocity has a vertical component $v_{z}$ and a transverse component $R \omega$. In the experiments, the velocity due to the particle rotation $v_{\omega} \sim R \omega$ is negligible compared to the velocity of the fluid $v_{\omega} \ll v_{z}$. The scaling for this torque is given by

$$
\Gamma_{s} \sim R \times f \mathcal{S} \rho v_{z} v_{\omega} \sim f R^{4} \rho v_{z} \times \omega
$$

where $f$ is the friction factor, $\mathcal{S}$ is the surface of the sphere, $R$ its radius and $\rho$ the water density.

The friction factor is a dimensionless parameter analogous to the drag coefficient but for tangential flows with $f=0.316 \times R e^{-1 / 4}$ for pipe flows ${ }^{45,46}$. The scaling argument of Eq. (6) provides $\lambda=2 \times 10^{-7} \mathrm{~kg} \cdot \mathrm{m}^{2} \cdot \mathrm{s}^{-1}$ where

$$
\Gamma_{s}=-\lambda \omega
$$

In the limit of negligible thickness $e$, a point with coordinates $\{X, Y, Z\}$ lying on the surface of a helical tail aligned with the $z$-axis satisfies:

$$
\left(\begin{array}{c}
X \\
Y \\
Z
\end{array}\right)=\left(\begin{array}{c}
a \cos (h b-\psi(t)) \\
a \sin (h b-\psi(t)) \\
b
\end{array}\right)
$$

where $\{a, b\}$ are the surface coordinates with $-W / 2<$ $a<W / 2$ and $0<b<L$ (see figure 9). The set of coordinates $\{a, b\}$ relates to the cylindrical coordinates with $r=\sqrt{X^{2}+Y^{2}}=|a|$ and $Z=b . h$ is the twisting wavenumber of the helical surface or, equivalently, $\pi / h$ is its pitch. The phase $\psi(t)$ gives the angle of the helix with respect to time. The angular velocity of the rotating helix is given by $\omega(t)=d \psi(t) / d t$. A flat tail in the $X Z$ plane corresponds to $h=0$ and $\psi(t)=0$.

In the case of stationary rotation with angular velocity $\omega$, the tail profile (8) is invariant if $h b-\omega t=0$ and $b^{\prime}=b-(\omega / h) t$. The velocity $\omega / h$ is then a natural velocity for a rotation with angular frequency $\omega$ and twisting wavenumber $h$. If the natural velocity is the settling velocity of the flow $v_{s}$, the relation becomes $\omega_{s} / h=v_{s}$ where $\omega_{s}$ is the limit angular frequency used in equation (2).

The orientation of the surface is given by the vector obtained from the cross product between the derivatives of the surface coordinates with respect to the unit vector associated with $a$ and $b$ :

$$
\partial_{a}\left(\begin{array}{c}
X \\
Y \\
Z
\end{array}\right) \wedge \partial_{b}\left(\begin{array}{c}
X \\
Y \\
Z
\end{array}\right)=\left(\begin{array}{c}
\sin (h b-\psi(t)) \\
-\cos (h b-\psi(t)) \\
a h
\end{array}\right)
$$

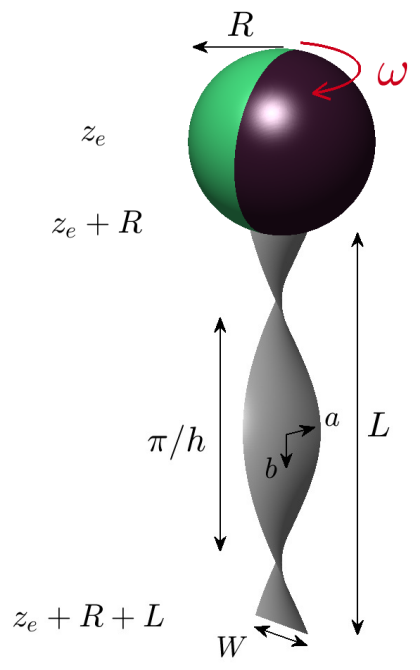

FIG. 9. Schematics of the model geometry used to describe the chiral particle. The sphere has a radius $R$ and the tail has a width $W$ and a length $L$. The set of coordinates $\{a, b\}$ is used as a parametrization for the surface of the helical tail. The pitch $\pi / h$ of the tail is represented in the figure where $h$ is the twisting wavenumber of the tail. The distance $z_{e}$ to the nozzle for the center of the sphere, the beginning of the tail $z_{e}+R$ and the end of the tail $z_{e}+R+L$ are also indicated.

The unit vector $\vec{n}$ defining the local orientation of the surface element is:

$$
\vec{n}=\frac{1}{\sqrt{1+h^{2} a^{2}}}\left(\begin{array}{c}
\sin (h b-\psi(t)) \\
-\cos (h b-\psi(t)) \\
a h
\end{array}\right)
$$

The net torque $\Gamma_{t}$ is obtained from the integration over the tail surface of a local stress computed from the local velocity $v_{z}$ established in equations (4) and (5) without a tail. The local velocity of the fluid with respect to the tail for a stationary rotation at $d \psi / d t=\omega_{e}$ is given by:

$$
\vec{v}=v_{z} \vec{z}+\partial_{t}\left(\begin{array}{c}
X \\
Y \\
Z
\end{array}\right)=\left(\begin{array}{c}
-a \omega_{e} \sin (h b-\psi(t)) \\
a \omega_{e} \cos (h b-\psi(t)) \\
-v_{z}
\end{array}\right)
$$

where $v_{z}=v_{j}\left(1+\sigma_{v}\right)$ is the empirical profile of the sphere wake where $\sigma_{v}$ is defined in equation (5).

The inclination angle $i$ between the velocity $\vec{v}$ and the normal $\vec{n}$ is considered to define the drag and the lift components $^{47}$ for a surface element:

$$
\begin{aligned}
d \vec{F}_{D} & =\frac{1}{2} c_{D}(i) d S \rho v^{2} \overrightarrow{u_{D}} \\
d \vec{F}_{L} & =\frac{1}{2} c_{L}(i) d S \rho v^{2} \overrightarrow{u_{L}}
\end{aligned}
$$

where $\rho$ is the density of water, $d S$ the surface element of the tail, $\overrightarrow{u_{D}}$ the unit vector aligned with the velocity 
$\vec{v}$ and $\overrightarrow{u_{L}}$ the unit vector perpendicular to $\vec{v}$ and in the plane $(\vec{v}, \vec{n})$.

The torque $\Gamma_{t}$ is then computed using the relation:

$$
\Gamma_{t}=\int_{(a, b)} \vec{r} \wedge\left(d \vec{F}_{D}+d \vec{F}_{L}\right)
$$

The solution for the stationary rotation of the helical particle is obtained when the total torque on the particle vanishes:

$$
\Gamma_{t}\left(\omega_{e}\right)-\lambda \omega_{e}=0
$$

where $\omega_{e}$ is the stationary angular frequency of the rotating particle.

The equation (15) is solved numerically and the stationary value $\omega_{e}$ is found after multiple iterations using a Newton's method approach. The two free parameters of the model are the damping coefficient $\lambda$ and the magnitude of the flow field $v_{0}=D /\left(z_{e}-z_{0}\right)$ with the trapping distance $z=z_{e}$ in eq. (3). The value $\lambda=2.0 \times 10^{-7}$ $\mathrm{kg} . \mathrm{m}^{2} \mathrm{~s}^{-1}$ is set from the scaling argument given in equation (6). The fitting function presented in figure 4 is with $v_{0}=0.85 \times v_{s}$, where $v_{s}$ is the settling velocity of the particle under gravity defined in equation (1) and the numerical value 0.85 is the only parameter effectively adjusted.

According to the numerical solution of equation (15), the efficiency of the rotation tends to zero when the tail length approaches zero. This is because the torque $\Gamma_{t}$ acting on the tail vanishes when the tail surface approaches zero.

In the limit of a negligible damping $\lambda=0$ and for a uniform flow $v_{s}$, the solution of equation (15) leads to $\omega_{e}=\omega_{s}$ where $\omega_{s}=h v_{s}$ is the optimal rotation frequency introduced in section III. This limit defines the optimal rotation regime with the efficiency $\eta=1$. For the chiral particles in the experiments, the efficiency reaches $20 \%$. The existence of friction and the reduced velocity in the wake of the sphere explain the limited performances for the efficiency of the chiral particles.

\section{CONCLUSION}

The levitation technique has been used to capture chiral particles in hydrodynamic levitation. The particles' tail are characterized by a uniform twisting wavenumber defined as a twisting angle per unit length. It was shown that this wavenumber is a control parameter that sets the rotation speed of the trapped particle. Consequently, the levitation technique can be used to infer the twist of a particle after a calibration of the relation between the twist and the angular rotation.

We have shown that a 3D-PTV technique can be used to characterize the stationary flow around a trapped sphere in a turbulent jet. Two representations are proposed: a first representation in the nozzle frame shows a scattering of the jet by the trapped sphere and a second representation in the sphere frame is used to investigate the wake. An empirical approach has been proposed to establish an explicit formulation for the velocity in the wake of the sphere.

The influence of the length of the tail on the rotation efficiency has been investigated. The experimental data are in good agreement with a torque model that involves the friction of the sphere, the drag and lift coefficients of the tail surface element and the velocity formulation for the wake behind the sphere proposed to this effect.

The levitation technique can be used to perform optimization with respect to physical parameters. Here, the length of the tail has been varied and the experiments and model show that the rotation rate is reaching an optimal value when the length of the tail is approaching 6 radii of the sphere. Other parameters could be optimized such as the value of the twisting parameter that maximizes the rotation for a tail of fixed length.

The performances of the chiral particles in rotation are estimated by a comparison with an optimal rotation regime without friction and for an equivalent uniform flow. The experimental rotation regime is always found below the optimal rotation regime. The fact that the rotation is proportional to the chirality magnitude is however not universal since some classes of non-chiral objects are able to exhibit stationary rotation. This is the case for the plant seeds that are self-rotating during a free fall $^{48-50}$. Other instabilities with free falling discs or free rising bubbles are also exhibiting particle rotation by a coupling with the self-established helical trajectories ${ }^{51}$. A perspective of this work would be to investigate the stability in a turbulent jet of such particles exhibiting self-rotation.

${ }^{1}$ R. D. Blevins, New York, Van Nostrand Reinhold Co., 1984, 568 p. (1984).

${ }^{2}$ D. J. Tritton, Physical fluid dynamics (Springer Science \& Business Media, 2012).

${ }^{3} \mathrm{~S}$. Tavoularis, Measurement in fluid mechanics (Cambridge University Press, 2005).

${ }^{4}$ E. Brandt, Science 243, 349 (1989).

${ }^{5}$ J. Feng and D. Joseph, Journal of Fluid Mechanics 315, 367 (1996).

${ }^{6}$ M. M. F. Saba, The Physics Teacher 35, 294 (1997).

${ }^{7} \mathrm{~S}$. Davoust and L. Jacquin, in TSFP DIGITAL LIBRARY ONLINE (Begel House Inc., 2009).

${ }^{8}$ T. López-Arias, L. M. Gratton, G. Zendri, and S. Oss, Physics Education 46, 146 (2011).

${ }^{9}$ N. Mordant and J.-F. Pinton, The European Physical Journal B-Condensed Matter and Complex Systems 18, 343 (2000).

${ }^{10}$ P. Bagchi and S. Balachandar, Physics of fluids 15, 3496 (2003).

${ }^{11}$ H. Homann, J. Bec, and R. Grauer, Journal of Fluid Mechanics 721, 155 (2013).

12 R. Ojha, P.-A. Lemieux, P. Dixon, A. Liu, and D. Durian, Nature 427, 521 (2004).

${ }^{13}$ C. Cohen, B. Darbois-Texier, G. Dupeux, E. Brunel, D. Quéré, and C. Clanet, Proceedings of the Royal Society A: Mathematical, Physical and Engineering Sciences 470, 20130497 (2014).

${ }^{14}$ C. Cummins, M. Seale, A. Macente, D. Certini, E. Mastropaolo, I. M. Viola, and N. Nakayama, Nature 562, 414 (2018).

${ }^{15}$ D. Fabre, J. Tchoufag, V. Citro, F. Giannetti, and P. Luchini, Theoretical and Computational Fluid Dynamics 31, 475 (2017).

${ }^{16}$ A. J. Adler, B. J. Boyle, and F. Mandelbaum, "Football with fins," (1993), uS Patent 5,269,514. 
${ }^{17}$ J. D. Myers, "Toy football with spiral like tail," (2000), uS Patent 6,120,398.

${ }^{18}$ J. Rabault, R. A. Fauli, and A. Carlson, Physical review letters 122, 024501 (2019).

${ }^{19}$ S. Farhadi, S. Machaca, J. Aird, B. O. T. Maldonado, S. Davis, P. E. Arratia, and D. J. Durian, Soft matter 14, 5588 (2018).

${ }^{20}$ B. A. Grzybowski, H. A. Stone, and G. M. Whitesides, Nature 405, 1033 (2000).

${ }^{21}$ E. Climent, K. Yeo, M. R. Maxey, and G. E. Karniadakis, Journal of fluids engineering 129, 379 (2007).

${ }^{22}$ N. Uchida and R. Golestanian, Physical review letters 104, 178103 (2010).

${ }^{23}$ M. Leoni and T. Liverpool, EPL (Europhysics Letters) 92, 64004 (2011).

${ }^{24}$ K. Yeo, E. Lushi, and P. M. Vlahovska, Physical review letters 114, 188301 (2015).

${ }^{25}$ R. Wille and A. Fernholz, J. Fluid Mech. 23, 801 (1965).

${ }^{26}$ T. A. Vil'gel'mi, Journal of Applied Mechanics and Technical Physics 10, 76 (1969).

${ }^{27}$ R. Zimmermann, Y. Gasteuil, M. Bourgoin, R. Volk, A. Pumir, and J.-F. Pinton, Physical review letters 106, 154501 (2011).

${ }^{28} \mathrm{~S}$. F. Hoerner, Fluid-dynamic drag: practical information on aerodynamic drag and hydrodynamic resistance (Hoerner Fluid Dynamics, 1965).

${ }^{29}$ U. Lācis, S. Olivieri, A. Mazzino, and S. Bagheri, Physical Review Fluids 2, 033901 (2017).

${ }^{30}$ L. Lu, X.-l. Guo, G.-q. Tang, M.-m. Liu, C.-q. Chen, and Z.-h. Xie, Physics of Fluids 28, 093604 (2016)

${ }^{31} \mathrm{Y}$. Yokoi, in Proceedings of the 5th International Conference on Jets, Wakes and Separated Flows (ICJWSF2015) (Springer, 2016) pp. 181-187.

${ }^{32}$ D. Doh, T. Hwang, and T. Saga, Measurement Science and Technology 15, 1059 (2004).

${ }^{33}$ C. J. Kähler, S. Scharnowski, and C. Cierpka, Experiments in fluids 52, 1641 (2012)

${ }^{34}$ H. Schlichting, K. Gersten, E. Krause, H. Oertel, and K. Mayes, Boundary-layer theory, Vol. 7 (Springer, 1955).

${ }^{35}$ I. Wygnanski and H. Fiedler, Journal of Fluid Mechanics 38, 577 (1969).

${ }^{36}$ S. B. Pope, Turbulent Flows (Cambridge University Press, 2000).

${ }^{37}$ H. Reichardt, The Aeronautical Journal 47, 167 (1943).

${ }^{38}$ L. G. Alexander, T. Baron, and E. W. Comings, "Transport of momentum, mass, and heat in turbulent jets," Tech. Rep. (University of Illinois at Urbana Champaign, College of Engineering ..., 1953)

${ }^{39}$ H. J. Hussein, S. P. Capp, and W. K. George, Journal of Fluid Mechanics 258, 31 (1994).

${ }^{40}$ P. C. Chu, J. H. Lee, and V. H. Chu, Journal of Hydraulic Engineering 125, 193 (1999).

${ }^{41}$ K. T. McDonald, American Journal of Physics 68, 388 (2000).

${ }^{42} \mathrm{G}$. Xu and R. Antonia, Experiments in Fluids 33, 677 (2002).

${ }^{43}$ S. J. Kwon and I. W. Seo, Experiments in fluids 38, 801 (2005).

${ }^{44}$ T. Barois, P. Huck, M. Bourgoin, and R. Volk, Physical Review E 96, 033105 (2017).

${ }^{45} \mathrm{H}$. Blasius, in Mitteilungen über Forschungsarbeiten auf dem Gebiete des Ingenieurwesens, Vol. 134 (Springer, 1913) pp. 1-41.

${ }^{46} \mathrm{~J}$. Nikuradse, Laws of flow in rough pipes (National Advisory Committee for Aeronautics Washington, 1950).

${ }^{47}$ X. Ortiz, D. Rival, and D. Wood, Energies 8, 2438 (2015).

${ }^{48}$ A. Azuma and K. Yasuda, Journal of Theoretical Biology 138, 23 (1989)

${ }^{49}$ D. Lentink, W. B. Dickson, J. L. Van Leeuwen, and M. H. Dickinson, Science 324, 1438 (2009).

${ }^{50}$ K. Varshney, S. Chang, and Z. J. Wang, Nonlinearity 25, C1 (2011).

${ }^{51}$ P. Ern, F. Risso, D. Fabre, and J. Magnaudet, Annual Review of Fluid Mechanics 44, 97 (2012). 